\title{
OVERALL SURVIVAL AND DISEASE CONTROL RATES FOR OPERABLE INVASIVE BREAST CANCER IN THE ERA OF CONSERVATIVE SURGERY; RETROSPECTIVE, INSTITUTIONAL, AND FIVE-YEAR FOLLOW-UP DATA
}

\author{
ANA CAR PETERKO ${ }^{1}$, MANUELA AVIROVIĆ², IVA SKOČILIĆ ${ }^{3}$, \\ PETRA VALKOVIĆ ZUJIĆ ${ }^{4}$ INGRID BELAC LOVASIĆ ${ }^{3}$ and FRANJO LOVASIĆ ${ }^{1}$ \\ ${ }^{1}$ Department of General Surgery and Surgical Oncology, Clinical Hospital Center Rijeka, Rijeka, Croatia; \\ ${ }^{2}$ University of Rijeka, Faculty of Medicine, Department of General Pathology and Pathologic Anatomy, \\ Rijeka, Croatia; \\ ${ }^{3}$ Department of Radiotherapy and Oncology, Clinical Hospital Center Rijeka, Rijeka, Croatia; \\ ${ }^{4}$ Department of Radiology, Clinical Hospital Center Rijeka, Rijeka, Croatia
}

\section{Summary}

Background: Following breast cancer treatment recommendations, the conservative approach is accepted and highly respected in the Clinical Hospital Center (CHC) Rijeka. However, we have found that institutional follow-up data are lacking. This retrospective analysis aims to update institutional data on survival and disease control rates.

Methods: From 2011 till 2014, 915 breast cancer patients underwent surgery at CHC Rijeka, and 615 were included in this analysis. The Institutional Ethics Committee approved the analysis.

Results: All patients were female, and the average age was 59 years. In the 5-year postoperative period, local, regional, and distant recurrence-free survival rates and overall survival and disease-free survival were calculated. All rates negatively correlate with a higher $\mathrm{T}$ and $\mathrm{N}$ status and a higher stage of the disease. The analysis has also demonstrated that in the pT1-3 pN0-1 subgroup, sentinel lymph node biopsy (SLNB) was not inferior to axillary lymph node dissection (ALND) in terms of locoregional control of disease and overall survival.

Conclusion: Besides updating institutional data, the analysis confirmed that overall survival and locoregional control of the disease in the upfront-surgery patients are similar between $\mathrm{pN} 0$ and $\mathrm{pN} 1$ subpopulations and between $\mathrm{pN} 2$ and $\mathrm{pN} 3$, but statistically significantly different between $\mathrm{pN} 0-1$ and $\mathrm{pN} 2-3$. Currently ongoing, prospective observational multicenter clinical trial aims to translate the significance of these results into the neoadjuvant era.

KEYWORDS: breast cancer, survival, recurrence

\section{BACKGROUND}

In the last few decades, there is a constant deescalation in the surgical approach to breast cancer (BC) patients. In all current guidelines, a conservative surgical approach is strongly recom-

Corresponding author: Ana Car Peterko, Department of General Surgery and Surgical Oncology, Clinical Hospital Center Rijeka, Krešimirova 42, 51000 Rijeka, Croatia.

e-mail: anacarpeterko@gmail.com mended for all early-stage BC patients. Following NSABP B-06 trial results (1), lumpectomy with whole breast radiation therapy (WBRT) became a standard of care for all T1-T2 BC patients, and due to the results of NSABP B-04 (2) and B-32 (3) trials, axillary lymph node dissection (ALND) was replaced with the sentinel lymph node biopsy (SLNB) in clinically node-negative (cN0) patients. Moreover, after the results of ACOSOG Z011 (4) and AMAROS (5) trial, ALND is no longer justi- 
fied, even in the case of pathologically node-positive $(\mathrm{pN}+)$ patients, considering that conservative breast surgery was made, WBRT would be conducted, and that no more than two nodes were found to be involved with metastasis. However, for the high-risk $\mathrm{pN}+$ patients, radiotherapy of the lymphatic drainage is highly recommended instead of ALND.

In Clinical Hospital Center (CHC), Rijeka, all mentioned recommendations are accepted into clinical practice and are highly respected in everyday work. However, in this era of conservative surgery, we have found that institutional and national follow-up data are lacking. Most of the results regarding survival and disease control currently come from randomized controlled trials, i.e., from the highly selected patients. The purpose of this retrospective analysis is to report our latest institutional updates on survival and disease control rates of unselected populations from the era of conservative surgical and adjuvant oncological approaches and to discuss the potential influence of these results translated into the present neoadjuvant era.

\section{PATIENTS AND METHODS}

From 2011 till 2014, 915 female BC patients were surgically treated in CHC Rijeka. However, we excluded patients older than 80 years or with M1 status at the time of surgery, recurrent, bilateral, or in situ disease, patients diagnosed with other malignant conditions, and those without follow-up data in the postoperative period. Therefore, 615 patients remained for the analysis. All data used for this analysis were extracted from the integrated hospital informatics system of CHC Rijeka with the approval of the Ethics Committee of CHC Rijeka.

Besides several T4 status patients, all patients were submitted to primary surgical treatment followed with adjuvant oncological systemic and locoregional treatment conducted according to the institutional protocol, i.e., according to the NCCN and St. Gallen's consensus guidelines for that period. In 283 cases (46.02\%), lumpectomy and SLNB were performed, in 127 (20.65\%) lumpectomy and ALND, in 34 (5.53\%) mastectomy and SLNB, in $164(26.99 \%)$ radical mastectomy, and for four patients $(0.65 \%)$ there was no data of surgical treat- ment type. Axillary surgery was guided by NSABP B-32 (3), ACOSOG Z010 (6), ACOSOG Z011 (4), and AMAROS (5) trial criteria.

Overall survival (OS) rate is defined as a rate of patients surviving in 5 postoperative years or the last recorded control date. Distant disease-free survival or progression-free survival (DDFS or PFS) rate is defined as a rate of patients without evidence of distant metastasis, i.e., M1 status in 5 postoperative years or at the date of the last recorded control. Local recurrence-free survival (LRFS) rate is defined as a rate of patients without evidence of disease in the already treated or contralateral breast or thoracic wall in the five postoperative years or at the date of the last recorded control. Regional recurrence-free survival (RRFS) rate is defined as a rate of patients without evidence of disease in the lymph nodes of unilateral or contralateral axilla, infraclavicular, supraclavicular, or internal mammary lymph nodes in 5 postoperative years or to the date of the last recorded control. Disease-free, i.e., progression-free survival (DFS) rate is defined as patients' rate without evidence of recurrence in 5 postoperative years or the date of the last recorded control. All rates are calculated by the method of Kaplan Meier. The results are analyzed using Statistica 13 software and interpreted at statistical significance $\mathrm{p}=0.05$. Clinical and pathological $\mathrm{T}, \mathrm{N}$, and stage of the disease are defined according to the 8th Edition of AJCC Cancer Staging Manual.

\section{RESULTS}

All patients were female, a mean age of 59 years at the time of the surgery and in stage T1-4 N0-3 M0 at the diagnosis time. The mean followup time was 56.5 months (+/- 10.8 months, range from 4 to 60 months). Study population characteristics and outcomes are displayed in Table 1.

Overall local, regional, and distant recurrence rates and overall survival and disease-free survival rates in 5 postoperative years are calculated by Kaplan Meier's method and displayed in Figure 1.

Furthermore, all rates are calculated in correlation with immunophenotype, $\mathrm{T}$ and $\mathrm{N}$ status, stage of the disease, and surgical treatment type. The results of these correlations are displayed in Table 2. 
Table 1.

Study population characteristics and the outcomes in terms of overall survival (OS), distant (DRFS), local (LRFS), and regional recurrence-free survival (RRFS) in the five years postoperatively.

\begin{tabular}{|c|c|c|c|c|c|c|c|c|c|c|c|c|c|c|c|}
\hline & \multirow{2}{*}{\multicolumn{2}{|c|}{ Patients }} & \multicolumn{3}{|c|}{ OS/5y } & \multicolumn{3}{|c|}{ DRFS/5y } & \multicolumn{3}{|c|}{ LRFS/5y } & \multicolumn{3}{|c|}{ RRFS/5y } \\
\hline & & & & \multirow{2}{*}{$\begin{array}{c}\text { Yes } \\
N(\%)\end{array}$} & \multirow{2}{*}{$\begin{array}{c}\text { No } \\
\mathrm{N}(\%)\end{array}$} & \multirow{2}{*}{$\begin{array}{c}\begin{array}{c}\text { No } \\
\text { data }\end{array} \\
\mathrm{N}(\%)\end{array}$} & \multirow{2}{*}{$\begin{array}{c}\text { Yes } \\
\mathrm{N}(\%)\end{array}$} & \multirow{2}{*}{$\begin{array}{c}\text { No } \\
\mathrm{N}(\%)\end{array}$} & \multirow{2}{*}{$\begin{array}{c}\begin{array}{c}\text { No } \\
\text { data }\end{array} \\
\mathrm{N}(\%)\end{array}$} & \multirow{2}{*}{$\begin{array}{c}\text { Yes } \\
\mathrm{N}(\%)\end{array}$} & \multirow{2}{*}{$\begin{array}{c}\text { No } \\
\mathrm{N}(\%)\end{array}$} & \multirow{2}{*}{$\begin{array}{l}\begin{array}{c}\text { No } \\
\text { data }\end{array} \\
\mathrm{N}(\%)\end{array}$} & \multirow{2}{*}{$\begin{array}{c}\text { Yes } \\
\mathrm{N}(\%)\end{array}$} & \multirow{2}{*}{$\begin{array}{c}\text { No } \\
\mathrm{N}(\%)\end{array}$} & \multirow{2}{*}{$\begin{array}{c}\begin{array}{c}\text { No } \\
\text { data }\end{array} \\
\mathrm{N}(\%)\end{array}$} \\
\hline & & $\mathrm{N}$ & $\%$ & & & & & & & & & & & & \\
\hline \multirow{6}{*}{ 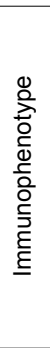 } & Luminal A & 227 & 36.91 & $\begin{array}{c}215 \\
(94.71)\end{array}$ & $\begin{array}{c}5 \\
(2.20)\end{array}$ & $\begin{array}{c}7 \\
(3.08)\end{array}$ & $\begin{array}{c}211 \\
(92.95)\end{array}$ & $\begin{array}{c}9 \\
(3.96)\end{array}$ & $\begin{array}{c}7 \\
(2.57)\end{array}$ & $\begin{array}{c}216 \\
(95.15)\end{array}$ & $\begin{array}{c}3 \\
(1.32)\end{array}$ & $\begin{array}{c}8 \\
(3.52)\end{array}$ & $\begin{array}{c}214 \\
(94.27) \\
\end{array}$ & $\begin{array}{c}5 \\
(2.20)\end{array}$ & $\begin{array}{c}8 \\
(3.52)\end{array}$ \\
\hline & Luminal B & 207 & 33.66 & $\begin{array}{c}185 \\
(89.37)\end{array}$ & $\begin{array}{c}3 \\
(1.45)\end{array}$ & $\begin{array}{c}19 \\
(9.17)\end{array}$ & $\begin{array}{c}172 \\
(83.09)\end{array}$ & $\begin{array}{c}5 \\
(2.41)\end{array}$ & $\begin{array}{c}10 \\
(4.83)\end{array}$ & $\begin{array}{c}184 \\
(88.88)\end{array}$ & $\begin{array}{c}10 \\
(4.83)\end{array}$ & $\begin{array}{c}13 \\
(6.28)\end{array}$ & $\begin{array}{c}182 \\
(87.92)\end{array}$ & $\begin{array}{c}8 \\
(3.86)\end{array}$ & $\begin{array}{c}17 \\
(8.21)\end{array}$ \\
\hline & Luminal B HER-2+ & 75 & 12.20 & $\begin{array}{c}65 \\
(86.66)\end{array}$ & $\begin{array}{c}5 \\
(6.67)\end{array}$ & $\begin{array}{c}5 \\
(6.67)\end{array}$ & $\begin{array}{c}64 \\
(85.33)\end{array}$ & $\begin{array}{c}6 \\
(8)\end{array}$ & $\begin{array}{c}5 \\
(6.66)\end{array}$ & $\begin{array}{c}68 \\
(90.66)\end{array}$ & $\begin{array}{c}2 \\
(2.66)\end{array}$ & $\begin{array}{c}5 \\
(6.67)\end{array}$ & $\begin{array}{c}67 \\
(89.33) \\
\end{array}$ & $\begin{array}{c}3 \\
(4)\end{array}$ & $\begin{array}{c}5 \\
(6.67)\end{array}$ \\
\hline & HER-2+ & 53 & 8.62 & $\begin{array}{c}38 \\
(71.69)\end{array}$ & $\begin{array}{c}8 \\
(15.09)\end{array}$ & $\begin{array}{c}7 \\
(13.21)\end{array}$ & $\begin{array}{c}36 \\
(67.92)\end{array}$ & $\begin{array}{c}12 \\
(22.64)\end{array}$ & $\begin{array}{c}5 \\
(9.43)\end{array}$ & $\begin{array}{c}41 \\
(77.36)\end{array}$ & $\begin{array}{c}7 \\
(13.21)\end{array}$ & $\begin{array}{c}5 \\
(9.43)\end{array}$ & $\begin{array}{c}42 \\
(79.25) \\
\end{array}$ & $\begin{array}{c}5 \\
(9.43)\end{array}$ & $\begin{array}{c}6 \\
(11.32)\end{array}$ \\
\hline & TNBC & 48 & 7.80 & $\begin{array}{c}34 \\
(70.83) \\
\end{array}$ & $\begin{array}{c}1 \\
(2.08) \\
\end{array}$ & $\begin{array}{c}13 \\
(27.08) \\
\end{array}$ & $\begin{array}{c}33 \\
(68.75) \\
\end{array}$ & $\begin{array}{c}8 \\
(16.67) \\
\end{array}$ & $\begin{array}{c}7 \\
(14.58) \\
\end{array}$ & $\begin{array}{c}34 \\
(70.83) \\
\end{array}$ & $\begin{array}{c}8 \\
(16.67) \\
\end{array}$ & $\begin{array}{c}6 \\
(12.5)\end{array}$ & $\begin{array}{c}36 \\
(75) \\
\end{array}$ & $\begin{array}{c}2 \\
(4.17)\end{array}$ & $\begin{array}{c}10 \\
(20.83)\end{array}$ \\
\hline & Unknown & 5 & 0.81 & $\begin{array}{c}2 \\
(40)\end{array}$ & $\begin{array}{c}3 \\
(60)\end{array}$ & 0 & $\begin{array}{c}2 \\
(40)\end{array}$ & $\begin{array}{c}1 \\
(20) \\
\end{array}$ & $\begin{array}{c}2 \\
(40)\end{array}$ & $\begin{array}{c}3 \\
(60) \\
\end{array}$ & 0 & $\begin{array}{c}2 \\
(40)\end{array}$ & $\begin{array}{c}3 \\
(60)\end{array}$ & 0 & $\begin{array}{c}2 \\
(40)\end{array}$ \\
\hline & & & & & & & & & & & & & & & \\
\hline \multirow{5}{*}{ 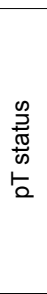 } & T1 & 350 & 56.91 & $\begin{array}{c}330 \\
(94.29) \\
\end{array}$ & $\begin{array}{c}3 \\
(0.86)\end{array}$ & $\begin{array}{c}17 \\
(4.86) \\
\end{array}$ & $\begin{array}{c}328 \\
(93.71) \\
\end{array}$ & $\begin{array}{c}12 \\
(3.43) \\
\end{array}$ & $\begin{array}{c}10 \\
(2.86)\end{array}$ & $\begin{array}{l}329 \\
(94) \\
\end{array}$ & $\begin{array}{c}12 \\
(3.43)\end{array}$ & $\begin{array}{c}9 \\
(2.57)\end{array}$ & $\begin{array}{c}330 \\
(94.29) \\
\end{array}$ & $\begin{array}{c}8 \\
(2.29) \\
\end{array}$ & $\begin{array}{c}12 \\
(3.43)\end{array}$ \\
\hline & $\mathrm{T} 2$ & 195 & 31.71 & $\begin{array}{c}162 \\
(83.08)\end{array}$ & $\begin{array}{c}13 \\
(6.67)\end{array}$ & $\begin{array}{c}20 \\
(10.26)\end{array}$ & $\begin{array}{c}148 \\
(75.90)\end{array}$ & $\begin{array}{c}32 \\
(16.41)\end{array}$ & $\begin{array}{c}15 \\
(7.70)\end{array}$ & $\begin{array}{c}170 \\
(87.18)\end{array}$ & $\begin{array}{c}8 \\
(4.11)\end{array}$ & $\begin{array}{c}17 \\
(8.72)\end{array}$ & $\begin{array}{c}165 \\
(84.62)\end{array}$ & $\begin{array}{c}8 \\
(4.10)\end{array}$ & $\begin{array}{c}22 \\
(11.28)\end{array}$ \\
\hline & T3 & 25 & 4.07 & $\begin{array}{c}21 \\
(84)\end{array}$ & $\begin{array}{c}3 \\
(12) \\
\end{array}$ & $\begin{array}{c}1 \\
(4)\end{array}$ & $\begin{array}{c}19 \\
(76)\end{array}$ & $\begin{array}{c}4 \\
(16)\end{array}$ & $\begin{array}{c}2 \\
(8)\end{array}$ & $\begin{array}{c}20 \\
(80)\end{array}$ & $\begin{array}{c}3 \\
(12) \\
\end{array}$ & $\begin{array}{c}2 \\
(8)\end{array}$ & $\begin{array}{c}21 \\
(84)\end{array}$ & \begin{tabular}{|c|}
1 \\
$(4)$ \\
\end{tabular} & $\begin{array}{c}3 \\
(12) \\
\end{array}$ \\
\hline & T4 & 42 & 6.83 & $\begin{array}{c}26 \\
(61.91)\end{array}$ & $\begin{array}{c}3 \\
(7.14)\end{array}$ & $\begin{array}{c}13 \\
(30.95) \\
\end{array}$ & $\begin{array}{c}23 \\
(54.76) \\
\end{array}$ & $\begin{array}{c}13 \\
(30.95)\end{array}$ & $\begin{array}{c}6 \\
(14.29) \\
\end{array}$ & $\begin{array}{c}27 \\
(64.29) \\
\end{array}$ & $\begin{array}{c}7 \\
(16.67)\end{array}$ & $\begin{array}{c}8 \\
(19.05) \\
\end{array}$ & $\begin{array}{c}28 \\
(59.52) \\
\end{array}$ & $\begin{array}{c}6 \\
(14.29) \\
\end{array}$ & $\begin{array}{c}8 \\
(19.05)\end{array}$ \\
\hline & Unknown & 3 & 0.49 & 0 & $\begin{array}{c}3 \\
(100)\end{array}$ & 0 & 0 & 0 & $\begin{array}{c}3 \\
(100)\end{array}$ & 0 & 0 & $\begin{array}{c}3 \\
(100)\end{array}$ & 0 & 0 & $\begin{array}{c}3 \\
(100)\end{array}$ \\
\hline & & & & & & & & & & & & & & & \\
\hline \multirow{5}{*}{$\begin{array}{l}\frac{\infty}{P} \\
\frac{1}{\pi} \\
\frac{1}{\infty} \\
z \\
0\end{array}$} & NO & 371 & 60.33 & $\begin{array}{c}343 \\
(92.45) \\
\end{array}$ & $\begin{array}{c}7 \\
/ 1.89) \\
\end{array}$ & $\begin{array}{c}21 \\
(5.67)\end{array}$ & $\begin{array}{c}343 \\
(92.45) \\
\end{array}$ & $\begin{array}{c}14 \\
(3.77)\end{array}$ & $\begin{array}{c}14 \\
(3.77)\end{array}$ & $\begin{array}{c}343 \\
(92.45)\end{array}$ & $\begin{array}{c}14 \\
(3.77)\end{array}$ & $\begin{array}{c}14 \\
(3.77)\end{array}$ & \begin{tabular}{|c|}
345 \\
$(92.99)$ \\
\end{tabular} & $\begin{array}{c}8 \\
(2.16) \\
\end{array}$ & $\begin{array}{c}18 \\
(4.85)\end{array}$ \\
\hline & N1 & 148 & 24.07 & $\begin{array}{c}135 \\
(91.22) \\
\end{array}$ & $\begin{array}{c}3 \\
(2.03)\end{array}$ & $\begin{array}{c}10 \\
(6.76)\end{array}$ & $\begin{array}{c}124 \\
(83.78) \\
\end{array}$ & $\begin{array}{c}17 \\
(11.49)\end{array}$ & $\begin{array}{c}7 \\
(4.73)\end{array}$ & $\begin{array}{c}135 \\
(91.22) \\
\end{array}$ & $\begin{array}{c}5 \\
(3.38)\end{array}$ & $\begin{array}{c}8 \\
(5.41)\end{array}$ & $\begin{array}{c}135 \\
(91.22 \\
\end{array}$ & $\begin{array}{c}3 \\
(2.02)\end{array}$ & $\begin{array}{c}10 \\
(6.76)\end{array}$ \\
\hline & N2 & 58 & 9.43 & $\begin{array}{c}38 \\
(65.51)\end{array}$ & $\begin{array}{c}6 \\
(10.34) \\
\end{array}$ & $\begin{array}{c}14 \\
(24.14) \\
\end{array}$ & $\begin{array}{c}32 \\
(55.17) \\
\end{array}$ & $\begin{array}{c}21 \\
(36.21)\end{array}$ & $\begin{array}{c}5 \\
(8.62)\end{array}$ & $\begin{array}{c}41 \\
(70.69) \\
\end{array}$ & $\begin{array}{c}7 \\
(12.07) \\
\end{array}$ & $\begin{array}{c}10 \\
(17.24) \\
\end{array}$ & $\begin{array}{c}40 \\
68.97)\end{array}$ & $\begin{array}{c}7 \\
(12.07) \\
\end{array}$ & $\begin{array}{c}11 \\
(18.97)\end{array}$ \\
\hline & N3 & 35 & 5.69 & $\begin{array}{c}23 \\
(65.71)\end{array}$ & $\begin{array}{c}6 \\
(17.14)\end{array}$ & $\begin{array}{c}6 \\
(17.14)\end{array}$ & $\begin{array}{c}19 \\
(54.29) \\
\end{array}$ & $\begin{array}{c}9 \\
(25.71)\end{array}$ & $\begin{array}{c}7 \\
(20)\end{array}$ & $\begin{array}{c}27 \\
(77.14)\end{array}$ & $\begin{array}{c}4 \\
(11.43)\end{array}$ & $\begin{array}{c}4 \\
(11.43)\end{array}$ & $\begin{array}{c}24 \\
(68.57)\end{array}$ & $\begin{array}{c}5 \\
(14.29) \\
\end{array}$ & $\begin{array}{c}6 \\
(17.14)\end{array}$ \\
\hline & Unknown & 3 & 0.49 & 0 & $\begin{array}{c}3 \\
(100) \\
\end{array}$ & 0 & 0 & 0 & $\begin{array}{c}3 \\
(100) \\
\end{array}$ & 0 & 0 & $\begin{array}{c}3 \\
(100) \\
\end{array}$ & 0 & 0 & $\begin{array}{c}3 \\
(100)\end{array}$ \\
\hline & & & & & & & & & & & & & & & \\
\hline \multirow{8}{*}{$\begin{array}{l}\underset{\mathbb{D}}{\mathbb{D}} \\
\underset{\infty}{\infty} \\
\sum_{1}\end{array}$} & IA & 263 & 42.76 & $\begin{array}{c}250 \\
(95.06)\end{array}$ & $\begin{array}{c}2 \\
(0.76)\end{array}$ & $\begin{array}{c}11 \\
(4.18)\end{array}$ & $\begin{array}{c}250 \\
(95.06)\end{array}$ & $\begin{array}{c}6 \\
(0.23)\end{array}$ & $\begin{array}{c}7 \\
(2.66)\end{array}$ & $\begin{array}{c}249 \\
(94.68)\end{array}$ & $\begin{array}{c}6 \\
(2.28)\end{array}$ & $\begin{array}{c}8 \\
(3.04)\end{array}$ & $\begin{array}{c}249 \\
(94.68)\end{array}$ & $\begin{array}{c}5 \\
(1.90)\end{array}$ & $\begin{array}{c}9 \\
(3.42)\end{array}$ \\
\hline & IB & 20 & 3.25 & $\begin{array}{c}20 \\
(100)\end{array}$ & 0 & 0 & $\begin{array}{c}20 \\
(100)\end{array}$ & 0 & 0 & $\begin{array}{c}20 \\
(20)\end{array}$ & 0 & 0 & $\begin{array}{c}20 \\
(100)\end{array}$ & 0 & 0 \\
\hline & IIA & 144 & 23.41 & $\begin{array}{c}129 \\
(89.58)\end{array}$ & $\begin{array}{c}4 \\
(2.78)\end{array}$ & $\begin{array}{c}11 \\
(7.64)\end{array}$ & $\begin{array}{c}125 \\
(86.81) \\
\end{array}$ & $\begin{array}{c}11 \\
(7.64)\end{array}$ & $\begin{array}{c}8 \\
(5.56)\end{array}$ & $\begin{array}{c}128 \\
(88.89)\end{array}$ & $\begin{array}{c}11 \\
(7.64)\end{array}$ & $\begin{array}{c}5 \\
(3.47)\end{array}$ & $\begin{array}{c}129 \\
(89.58) \\
\end{array}$ & $\begin{array}{c}5 \\
(3.47) \\
\end{array}$ & $\begin{array}{c}10 \\
(6.94)\end{array}$ \\
\hline & IIB & 60 & 9.76 & $\begin{array}{c}52 \\
(86.67) \\
\end{array}$ & $\begin{array}{c}3 \\
(5)\end{array}$ & $\begin{array}{c}5 \\
(8.33) \\
\end{array}$ & $\begin{array}{c}46 \\
(76.67) \\
\end{array}$ & $\begin{array}{c}10 \\
(16.67) \\
\end{array}$ & $\begin{array}{c}4 \\
(6.67)\end{array}$ & $\begin{array}{c}54 \\
(90) \\
\end{array}$ & $\begin{array}{c}5 \\
(8.33)\end{array}$ & $\begin{array}{c}6 \\
(10)\end{array}$ & $\begin{array}{c}53 \\
(88.33) \\
\end{array}$ & $\begin{array}{c}1 \\
(1.17)\end{array}$ & $\begin{array}{c}6 \\
(10)\end{array}$ \\
\hline & IIIA & 56 & 9.11 & $\begin{array}{c}45 \\
(80.36)\end{array}$ & $\begin{array}{c}3 \\
(5.36) \\
\end{array}$ & $\begin{array}{c}8 \\
(41.29)\end{array}$ & $\begin{array}{c}40 \\
(71.43)\end{array}$ & $\begin{array}{c}13 \\
(23.21)\end{array}$ & $\begin{array}{c}3 \\
(5.36)\end{array}$ & $\begin{array}{c}46 \\
(82.14)\end{array}$ & $\begin{array}{c}3 \\
(5.36)\end{array}$ & $\begin{array}{c}5 \\
(8.93)\end{array}$ & $\begin{array}{c}47 \\
(83.93) \\
\end{array}$ & $\begin{array}{c}3 \\
(5.36)\end{array}$ & $\begin{array}{c}6 \\
(10.71)\end{array}$ \\
\hline & IIIB & 33 & 5.37 & $\begin{array}{c}19 \\
(57.58)\end{array}$ & $\begin{array}{c}5 \\
(15.15)\end{array}$ & $\begin{array}{c}9 \\
(27.27) \\
\end{array}$ & $\begin{array}{c}18 \\
(54.55) \\
\end{array}$ & $\begin{array}{c}10 \\
(30.30)\end{array}$ & $\begin{array}{c}5 \\
(15.15) \\
\end{array}$ & $\begin{array}{c}22 \\
(66.67) \\
\end{array}$ & $\begin{array}{c}5 \\
(15.15) \\
\end{array}$ & $\begin{array}{c}8 \\
(24.24) \\
\end{array}$ & $\begin{array}{c}22 \\
(66.67) \\
\end{array}$ & $\begin{array}{c}3 \\
(9.09)\end{array}$ & $\begin{array}{c}8 \\
(24.24)\end{array}$ \\
\hline & IIIC & 36 & 5.85 & $\begin{array}{c}24 \\
(66.67)\end{array}$ & $\begin{array}{c}5 \\
(13.89)\end{array}$ & $\begin{array}{c}7 \\
(19.44)\end{array}$ & $\begin{array}{c}19 \\
(52.78) \\
\end{array}$ & $\begin{array}{c}11 \\
(30.56)\end{array}$ & $\begin{array}{c}6 \\
(16.67) \\
\end{array}$ & $\begin{array}{c}27 \\
(75) \\
\end{array}$ & $\begin{array}{c}5 \\
(13.89) \\
\end{array}$ & $\begin{array}{c}4 \\
(11.11) \\
\end{array}$ & $\begin{array}{c}24 \\
(66.67) \\
\end{array}$ & $\begin{array}{c}6 \\
(16.67) \\
\end{array}$ & $\begin{array}{c}6 \\
(16.67) \\
\end{array}$ \\
\hline & Unknown & 3 & 0.49 & 0 & $\begin{array}{c}3 \\
(100)\end{array}$ & 0 & 0 & 0 & $\begin{array}{c}3 \\
(100) \\
\end{array}$ & 0 & 0 & $\begin{array}{c}3 \\
(100)\end{array}$ & 0 & 0 & $\begin{array}{c}3 \\
(100)\end{array}$ \\
\hline & & & & & & & & & & & & & & & \\
\hline \multirow{5}{*}{$\begin{array}{l}\frac{2}{0} \\
0 \\
= \\
\omega\end{array}$} & $B C S+S L N B$ & 284 & 46.18 & $\begin{array}{c}269 \\
(94.72) \\
\end{array}$ & $\begin{array}{c}2 \\
(0.70)\end{array}$ & $\begin{array}{c}13 \\
(4.56)\end{array}$ & $\begin{array}{c}268 \\
(94.37)\end{array}$ & $\begin{array}{c}8 \\
(2.82) \\
\end{array}$ & $\begin{array}{c}8 \\
(2.82)\end{array}$ & $\begin{array}{c}265 \\
(93.31)\end{array}$ & $\begin{array}{c}11 \\
(3.88)\end{array}$ & $\begin{array}{c}8 \\
(2.81) \\
\end{array}$ & $\begin{array}{c}268 \\
(94.37) \\
\end{array}$ & $\begin{array}{c}6 \\
(23.24) \\
\end{array}$ & $\begin{array}{c}10 \\
(3.52)\end{array}$ \\
\hline & BCS+ALND & 127 & 20.65 & $\begin{array}{c}116 \\
(91.34)\end{array}$ & $\begin{array}{c}4 \\
(3.15)\end{array}$ & $\begin{array}{c}7 \\
(5.51)\end{array}$ & $\begin{array}{c}111 \\
(87.40)\end{array}$ & $\begin{array}{c}12 \\
(9.45)\end{array}$ & $\begin{array}{c}4 \\
(3.15)\end{array}$ & $\begin{array}{c}116 \\
(91.34)\end{array}$ & $\begin{array}{c}8 \\
(6.3)\end{array}$ & $\begin{array}{c}3 \\
(2.36)\end{array}$ & $\begin{array}{c}118 \\
(92.91)\end{array}$ & $\begin{array}{c}2 \\
(1.57)\end{array}$ & $\begin{array}{c}7 \\
(5.51)\end{array}$ \\
\hline & M+SLNB & 34 & 5.53 & $\begin{array}{c}31 \\
(91.18) \\
\end{array}$ & $\begin{array}{c}1 \\
(2.94)\end{array}$ & $\begin{array}{c}2 \\
(5.88)\end{array}$ & $\begin{array}{c}31 \\
(91.18)\end{array}$ & $\begin{array}{c}1 \\
(2.94)\end{array}$ & $\begin{array}{c}2 \\
(5.88)\end{array}$ & $\begin{array}{c}32 \\
(94.12) \\
\end{array}$ & 0 & $\begin{array}{c}2 \\
(5.88) \\
\end{array}$ & $\begin{array}{c}32 \\
(94.12) \\
\end{array}$ & 0 & $\begin{array}{c}2 \\
(5.88)\end{array}$ \\
\hline & M+ALND & 166 & 26.99 & $\begin{array}{c}122 \\
(73.49)\end{array}$ & $\begin{array}{c}15 \\
(9.04)\end{array}$ & $\begin{array}{c}29 \\
(17.47)\end{array}$ & $\begin{array}{c}107 \\
(64.46)\end{array}$ & $\begin{array}{c}40 \\
(24.1)\end{array}$ & $\begin{array}{c}19 \\
(11.45)\end{array}$ & $\begin{array}{c}132 \\
(79.52)\end{array}$ & $\begin{array}{c}11 \\
(6.63)\end{array}$ & $\begin{array}{c}23 \\
(13.86)\end{array}$ & $\begin{array}{c}125 \\
(75.30) \\
\end{array}$ & $\begin{array}{c}15 \\
(9.04)\end{array}$ & $\begin{array}{c}26 \\
(15.66)\end{array}$ \\
\hline & Unknown & 4 & 0.65 & $\begin{array}{c}1 \\
(25)\end{array}$ & $\begin{array}{c}3 \\
(75)\end{array}$ & 0 & $\begin{array}{c}1 \\
(25)\end{array}$ & 0 & $\begin{array}{c}3 \\
(75)\end{array}$ & $\begin{array}{c}1 \\
(25)\end{array}$ & 0 & $\begin{array}{c}3 \\
(75)\end{array}$ & $\begin{array}{c}1 \\
(25)\end{array}$ & 0 & $\begin{array}{c}3 \\
(75)\end{array}$ \\
\hline
\end{tabular}

Table Legend: Immunophenotype is defined according to 2013 St. Gallen consensus [7], TNBC=Triple negative breast cancer, N=number, OS/5y=5 years overall survival, $D R F S / 5 y=5$ years disease-free survival, $L R F S / 5 y=5$ years local recurrence-free survival, RRFS/5y $=5$ years regional recurrence-free survival, pathological $T, N$ and stage of the disease are defined according to current TNM classification by $8^{\text {th }}$ Edition AJCC Cancer Staging Manual, BCS=breast conserving surgery (lumpectomy), SLNB=sentinel lymph node biopsy, M=mastectomy and $A L N D=$ axillary lymph node dissection. 

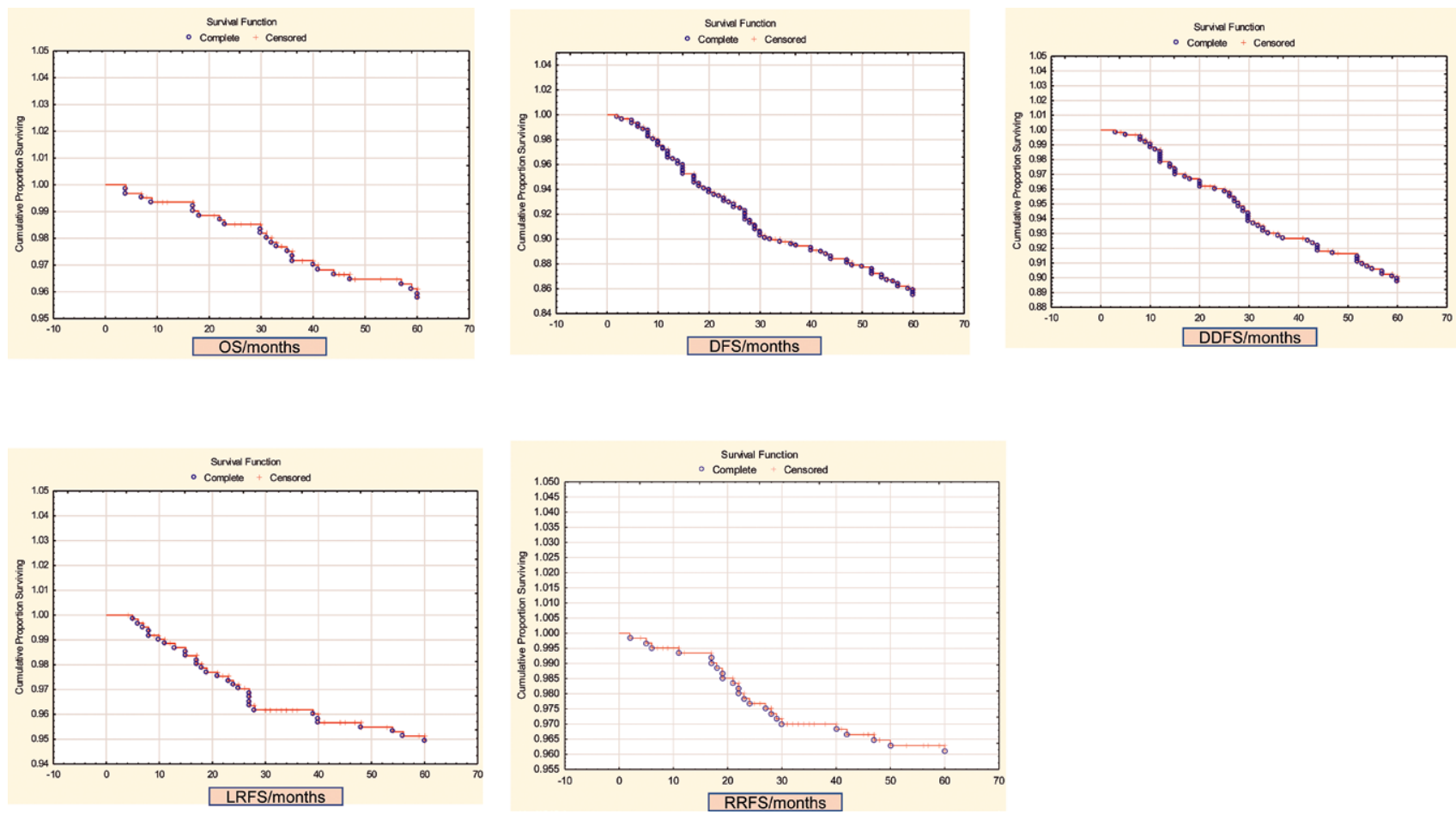

Figure 1.5 years overall survival rate (95.93\%), disease-free survival rate (85.85\%), distant disease-free survival rate (90.08\%), local recurrence-free survival rate (95.12\%), and regional recurrence-free survival rate $(96.26 \%)$ for the whole cohort, calculated by the method of Kaplan Meier

Table 2.

Correlation of survival rates with immunophenotype, $T, N$, and stage of the disease are calculated, and the test results (Chi-square) with the associated $p$-values are displayed for each correlation.

\begin{tabular}{|l|l|l|l|l|l|l|}
\hline & Test statistic & OS & DFS & DDFS & LRFS & RRFS \\
\hline \multirow{3}{*}{ Immunophenotype } & Chi-square & 27.051 & 37.359 & 26.978 & 30.577 & 7.062 \\
\cline { 2 - 7 } & p-value & 0.00002 & 0.00000 & 0.00003 & 0.00000 & 0.13260 \\
\hline \multirow{3}{*}{ T status } & Chi-square & 20.418 & 49.996 & 50.579 & 17.095 & 16.036 \\
\cline { 2 - 7 } & p-value & 0.00004 & 0.00000 & 0.00000 & 0.00068 & 0.00112 \\
\hline \multirow{2}{*}{ N status } & Chi-square & 30.050 & 73.528 & 72.197 & 12.319 & 27.290 \\
\cline { 2 - 7 } & p-value & 0.00000 & 0.00000 & 0.00000 & 0.00637 & 0.00001 \\
\hline \multirow{2}{*}{ Stage of disease } & Chi-square & 34.679 & 73.267 & 70.774 & 20.419 & 25.838 \\
\cline { 2 - 7 } & p-value & 0.00001 & 0.00000 & 0.00000 & 0.00234 & 0.00024 \\
\hline
\end{tabular}

Except for the RRFS, all rates are statistically significantly correlated to cancer immunophenotype as well as with $T$ status, $N$ status, and the stage of the disease.

As expected, survival rates are decreasing with the higher $\mathrm{T}$ and $\mathrm{N}$ status on the pathology report and a higher stage of the disease. However, OS, LRFS, and RRFS rates are not statistically significantly different between $\mathrm{pN} 0$ and $\mathrm{pN} 1$ and between $\mathrm{pN} 2$ and $\mathrm{pN} 3$, but among these two groups, the statistically significant difference is recorded.

All rates are also calculated for the pT1-3 pN0-1 subgroup concerning the extent of axillary surgery, demonstrating that SLNB is not inferior to ALND in terms of disease control (DFS, DDFS, LRFS, RRFS) and overall survival for this subpopulation of $\mathrm{BC}$ patients.

\section{DISCUSSION AND CONCLUSION}

Development of novel anticancer drugs and increasing trend of neoadjuvant oncological ap- 
Table $3 a$ and $3 b$ :

Overall survival, local recurrence-free survival, and regional recurrence-free survival according to the N status; between N0 and N1, as well as between N2 and N3, we have found no statistically significant differences (3a), but we have found a significant difference in prognosis between N0-1 and N2-3 (3b).

\begin{tabular}{|l|l|l|l|l|}
\hline Table 3a & N0 & N1 & N2 & N3 \\
\hline OS & $98.11 \%$ & $97.97 \%$ & $89.66 \%$ & $82.86 \%$ \\
\hline LRFS & $96.23 \%$ & $96.62 \%$ & $87.93 \%$ & $88.57 \%$ \\
\hline RRFS & $97.84 \%$ & $97.97 \%$ & $87.93 \%$ & $85.71 \%$ \\
\hline
\end{tabular}

\begin{tabular}{|l|l|l|l|l|}
\hline Table 3b & N0-N1 & N2-N3 & Log-Rank test & p-value \\
\hline OS & $98.07 \%$ & $87.10 \%$ & -5.39 & 0.00000 \\
\hline LRFS & $96.34 \%$ & $88.17 \%$ & -3.50 & 0.00046 \\
\hline RRFS & $97.88 \%$ & $87.10 \%$ & -5.17 & 0.00000 \\
\hline
\end{tabular}

Table 4.

Survival rates for T1-3 N0-1 subpopulation correlate to the extent of axillary surgery; the extent of axillary surgery is not related to overall survival and locoregional control of the disease in this subpopulation of breast cancer patients.

\begin{tabular}{|l|l|l|l|l|}
\hline & SLNB & ALND & Log-Rank test & p-value \\
\hline OS & $99.04 \%$ & $96.11 \%$ & -2.27 & 0.02305 \\
\hline DFS & $93.93 \%$ & $86.67 \%$ & -2.79 & 0.00520 \\
\hline DDFS & $97.12 \%$ & $90.00 \%$ & -3.43 & 0.00060 \\
\hline LRFS & $96.49 \%$ & $96.67 \%$ & 0.05 & 0.95813 \\
\hline RRFS & $98.08 \%$ & $97.22 \%$ & -0.67 & 0.50231 \\
\hline
\end{tabular}

proach for operable $\mathrm{BC}$ are leading to even less surgery. Several ongoing trials $(8,9,10)$ aim for more de-escalation of axillary surgery for early BC patients in the primary-surgery setting.

Besides updating our institutional data, we have demonstrated that pT4 status, pN2-3 status, and stage above IIIB are related to the worst oncological outcome in the primary surgery era. The analysis also indicates that $\mathrm{pN} 0$ and $\mathrm{pN} 1$ patients have similar outcomes in terms of OS and locoregional control of disease and the $\mathrm{pN} 2$ and $\mathrm{pN} 3 \mathrm{pa}-$ tients, but there is a statistically significant difference between $\mathrm{pN} 0-1$ and $\mathrm{pN} 2-3$ subgroups.

Moreover, according to our data, the extent of axillary surgery is not related to those outcomes in early and locally advanced (pT1-3 pN0-1) BC patients.

What might be the significance of these results translated in the neoadjuvant era? It is well known that preoperative systemic treatment does not affect OS and DFS for the whole cohort (11) but significantly improves the outcome of good responders to NAT.
The prospective observational multicenter trial, currently ongoing (12), is aiming to determine if there will be an improvement of survival rates of $\mathrm{cN} 2$ patients converted to $\mathrm{ycN} 0$ following NAT as well as the oncological safety of the ALND omission after SLNB, for initially cN1-2 patients converted to ypN0(sn) after NAT, in terms of overall survival and locoregional disease control.

Despite the reduced extent of axillary surgery, we do not expect worse oncological outcomes. Herein presented institutional data would provide the foundation for all anticipated comparations.

In addition, two ongoing randomized controlled trials, NEONOD II (13) and ATNEC (14), are yet another step ahead and are currently investigating if $\mathrm{cN} 1$ patients, converted to $\mathrm{ypN}+$ following NAT, but with minimal residual disease in the axilla, could be safely spared of ALND with or without radiotherapy to the lymphatic drainage, i.e., investigating if the results of ACOSOG Z010 (6), IBCSG 23-01 (15) and AMAROS (5) trial results can be safely translated into the neoadjuvant setting.

Although ypN+ signifies the resistance to the systemic treatment, based on our analysis, we strongly believe in the trials mentioned above' positive outcomes and in the safe translation of conservative axillary surgery into the neoadjuvant setting.

\section{Author Contributions:}

All authors contributed to the study conception and design. Ana Car Peterko and Manuela Avirović performed material preparation, data collection, and analysis. Ana Car Peterko wrote the first draft of the manuscript and all authors commented on previous versions of the manuscript. All authors read and approved the final manuscript.

\section{Statement of Ethics:}

The Ethics Committee approved this research of Clinical Hospital Center Rijeka. All procedures performed in studies involving human participants followed the institutional research committee's ethical standards and the 1964 Helsinki declaration and its later amendments or comparable ethical standards. For this type of study, formal consent is not required. This article does not con- 
tain any studies with animals performed by any of the authors.

Disclosure Statement: The authors have no conflict of interest to declare.

Funding Sources: none.

Acknowledgment: none

\section{REFERENCES}

1. Fisher B, Anderson S, Redmond CK, Wolmark N, Wickerham $L$ and Cronin WM. Reanalysis and results after 12 years of follow-up in a randomized clinical trial comparing total mastectomy with lumpectomy with or without irradiation in the treatment of breast cancer. N Engl J Med. 1995;333(20):1456-1461.

2. Fisher B, Montague E, Redmond C, Deutsch M, Brown GR, Zauber A et al. Findings from NSABP Protocol No. B-04-comparison of radical mastectomy with alternative treatments for primary breast cancer. I. Radiation compliance and its relation to treatment outcome. Cancer. 1980;46(1):1-13.

3. Krag DN, Anderson SJ, Julian TB, Brown AM, Harlow SP, Costantino JP et al. Sentinel-lymph-node resection compared with conventional axillary-lymph-node dissection in clinically node-negative patients with breast cancer: overall survival findings from the NSABP B-32 randomised phase 3 trial. Lancet Oncol. 2010;11(10):927-933.

4. Giuliano AE, Ballaman KV, Mc Call L, Beitsch PD, Brennan MB, Kelemen PR et al. Effect of Axillary Dissection vs No Axillary Dissection on 10-Year Overall Survival Among Women With Invasive Breast Cancer and Sentinel Node Metastasis The ACOSOG Z0011 (Alliance) Randomized Clinical Trial. JAMA. 2017;318 (10):918-926.

5. Donker M, van Tienhoven G, Straver ME, Meijnen P, van de Velde CJ, Mansel RE et al. Radiotherapy or surgery of the axilla after a positive sentinel node in breast cancer (EORTC 10981-22023 AMAROS): a randomised, multicentre, open-label, phase 3 non-inferiority trial. Lancet Oncol. 2014;15(12):1303-1310.

6. Giuliano AE, Hawes D, Ballman KV, Whitworth PW, Blumencranz PW, Reintgen DS, et al. Association of occult metastases in sentinel lymph nodes and bone marrow with survival among women with early-stage invasive breast cancer. JAMA. 2011;306:385-393.

7. Goldhirsch A, Winer EP, Coates AS, Gelber RD, Piccart-Gebhart $M$, Thürlimann B et al. Personalizing the treatment of women with early breast cancer: highlights of the St Gallen International Expert Consensus on the Primary Therapy of Early Breast Cancer. Annals of Oncology 2013; 24:2206-2223.
8. Gentilini $\mathrm{O}$, Veronesi. U: Abandoning sentinel lymph node biopsy in early breast cancer? A new trial in progress at the European Institute of Oncology of Milan (SOUND: Sentinel node vs Observation after axillary UltraSouND). Breast. 2012;21:678-681.

9. Reimer T, Stachs A, Nekljudova V, Loibl S, Hartmann $\mathrm{S}$, Wolter $\mathrm{K}$ et al. Restricted axillary staging in clinically and sonographically node-negative early invasive breast cancer (c/iT1-2) in the context of breast conserving therapy: first results following commencement of the Intergroup-Sentinel-Mamma (INSEMA) trial. Geburtsh Frauenheilk 2017;77:149-157.

10. Van Roozendaal LM, Vane MLG, van Dalen T, van der Hage JA, Strobbe LJA, Boersma LJ et al. Clinically node negative breast cancer patients undergoing breast conserving therapy, sentinel lymph node procedure versus followup: a Dutch randomized controlled multicentre trial (BOOG 2013-08). BMC Cancer 2017;17:459.

11. Wolmark N, Wang J, Mamounas E, Bryant J and Fisher $B$. Preoperative chemotherapy in patients with operable breast cancer: nine-year results from National Surgical Adjuvant Breast and Bowel Project B-18. J Natl Cancer Inst Monogr 2001;30:96-102.

12. Car Peterko A, Avirović M, Mance D, Valković Zujić P, Belac Lovasić I and Lovasić F. Clinical impact of sentinel lymph node biopsy after neoadjuvant systemic treatment in Luminal B, HER-2 positive and triple negative breast cancer patients with initially involved axillary lymph node(s). Protocol for prospective, nonrandomised, observational clinical trial. Libri Oncol. 2019;47(1):29-34.

13. Tinterria C, Canavesea G, Bruzzib P, Dozinb B. NEONOD 2: Rationale and design of a multicenter non-inferiority trial to assess the effect of axillary surgery omission on the outcome of breast cancer patients presenting only micrometastasis in the sentinel lymph node after neoadjuvant chemotherapy. Contemp Clin Trials Commun. 2019;17:100496.

14. ClinicalTrials.gov [Internet]. Bethesda (MD): National Library of Medicine (US). 2000 Feb 29- Identifier: NCT04109079, Axillary Management in Breast Cancer Patients With Needle Biopsy Proven Nodal Metastases After Neoadjuvant Chemotherapy (ATNEC); 2019 Sep 30 [cited 2020 Mar 27]. Available form: https:// clinicaltrials.gov/ct2/show/record/NCT04109079

15. Galimberti V, Cole BF, Viale G, Veronesi P, Vicini E, Intra $\mathrm{M}$ et al. Axillary dissection vs.no axillary dissection in patients with cT1-T2 cN0 M0 breast cancer and micrometastases only in the sentinel node: ten-year results of the IBCSG 23-01 trial. Lancet Oncol. 2018; 19:1385-1393. 


\section{Sažetak \\ OPERABILNI INVAZIVNI KARCINOM DOJKE U ERI KONZERVATIVNE KIRURGIJE; RETROSPEKTIVNA ANALIZA 5-GODIŠNJEG PREŽIVLJENJA I KONTROLE BOLESTI}

\section{A. Car Peterko, M. Avirović, I. Skočilić, P. Valković Zujić, I. Belac Lovasić i F. Lovasić}

Uvod: Temeljem suvremenih preporuka za liječenje karcinoma dojke, u Kliničkom bolničkom centru (KBC) Rijeka prihvaća se i zagovara konzervativni kirurški pristup. Međutim, na razini naše institucije, ali i na nacionalnom nivou, malo je obrađenih rezultata liječenja. Cilj ove retrospektivne analize je ažuriranje institucionalnih podataka o preživljenju i kontroli bolesti.

Metode: U periodu od 2011. do 2014. godine, u KBC Rijeka operirano je 915 pacijentica oboljelih od karcinoma dojke, a njih 615 uključeno je u ovu analizu. Istraživanje je odobreno od Etičkog povjerenstva KBC Rijeka.

Rezultati: Sve su pacjentice bile žene, prosječno stare 59 godina. Izračunate su ukupne stope preživljenja bez lokalnog, regionalnog i udaljenog recidiva, te stope ukupnog preživljenja i preživljenja bez povrata bolesti u petogodišnjem postoperativnom periodu. Sve su stope u negativnoj korelaciji sa višim T i N statusom kao i višim stadijem bolesti. Osim navedenog, rezultati su potvrdili da je biopsija sentinel limfnog čvora ekvivalentna aksilarnoj disekciji u smislu lokoregionalne kontrole bolesti kao i ukupnog petogodišnjeg preživljenja u pT1-3 pN0-1 podskupini.

Zaključak: Osim ažuriranja podataka naše institucije, ova je analiza potvrdila da su stope preživljenja i lokoregionalne kontrole bolesti u eri primarnog kirurškog tretmana slične između pN0 i pN1 subpopulacija kao i među pN2 i pN3, međutim statistički značajno različite između pN0-1 I pN2-3 podskupina. Prospektivno, opservacijsko, multicentrično kliničko istraživanje koje je u tijeku pokušava istražiti značenje ovih rezultata u neoadjuvantoj eri.

KLJUČNE RIJEČI: karcinom dojke, preživljenje, recidiv 